\title{
Aneurisma do Tronco da Artéria Coronária Esquerda
}

\author{
José Ramos Filho', Otávio Carneiro de Andrade¹, Filipe Guilherme Giordano", \\ Diego de Oliveira Vilarinho ${ }^{1}$, Eveline Pagoto ${ }^{1}$, Jomora Custódio Ferreira ${ }^{1}$
}

\begin{abstract}
RESUMO
O aneurisma do tronco da artéria coronária esquerda é extremamente raro e resulta de doença coronariana grave, com poucos casos descritos na literatura. A mortalidade é elevada e a terapêutica ainda é controversa. Relatamos o caso de uma paciente de 61 anos, admitida no pronto-socorro de hospital universitário, com quadro clínico de síndrome coronariana aguda, cuja coronariografia demonstrou a presença de aneurisma gigante do tronco da artéria coronária esquerda. A paciente foi submetida a tratamento cirúrgico e permanece assintomática após um ano de acompanhamento ambulatorial.
\end{abstract}

DESCRITORES: Aneurisma coronário, cirurgia. Coronariopatia. Vasos coronários, cirurgia.

0 aneurisma de artéria coronária é uma afecção rara, com elevado potencial de complicações, e o aneurisma do tronco da artéria coronária esquerda é extremamente raro, com pouco mais de 30 casos descritos na literatura mundial ${ }^{1}$. Geralmente, os aneurismas das artérias coronárias são múltiplos e a aterosclerose coronariana é responsável por mais de $50 \%$ dos casos diagnosticados em adultos. Os locais mais acometidos são: as porções proximal e média da artéria coronária direita e a porção proximal das artérias coronárias descendente anterior e circunflexa $a^{2,3}$.

\section{RELATO DO CASO}

Mulher de 61 anos, branca, dona de casa, natural do interior do Estado de São Paulo, procurou atendimento no pronto-socorro de hospital universitário, em

\footnotetext{
1 Universidade São Francisco - Bragança Paulista, SP. Correspondência: José Ramos Filho. Rua Roterdam, 57 - Jardim Euroville - Bragança Paulista, SP - CEP 12917-040.

Tel.: (11) 4033-7494 • E-mail: jramos-uti@uol.com.br

Recebido em: 8/11/2007 • Aceito em: 15/4/2008
}

\section{SUMMARY}

Aneurysm of the Left Main Coronary Artery

Aneurysm in the coronary trunk is an uncommon angiographic finding, with few cases reported. The mortality is high and the therapeutics are still uncertain. The aneurysm of the left main coronary artery is a rare and the worse cause of coronary artery disease. We report a case of a 61-year-old-female patient admitted in the Hospital Emergency Unit with a history of acute coronary syndrome. Coronary angiography showed a giant aneurysm in the left main coronary artery. The patient was submitted to coronary artery bypass grafting and remains asymptomatic after a one-year follow-up.

DESCRIPTORS: Coronary aneurysm, surgery. Coronary disease. Coronary vessels, surgery.

17 de maio de 2006, com queixa de dor precordial irradiada para membro superior esquerdo, com duração de 20 minutos. A dor era do tipo queimação, acompanhada de náuseas, vômitos, sudorese fria, palpitações e desencadeada por esforço físico moderado, melhorando com repouso. O primeiro episódio ocorreu há 20 dias, com piora progressiva da intensidade nos últimos três dias, caracterizando angina instável.

Como antecedentes pessoais referia hipertensão arterial há dois anos, fazendo uso regular de medicamentos, não sabendo especificar quais medicamentos usava no momento da admissão. Ao exame físico observou-se pressão arterial de $170 \times 80 \mathrm{mmHg}$, freqüência cardíaca de 75 batimentos por minuto, ritmo cardíaco regular em dois tempos sem sopros, e ausculta pulmonar normal. Exames complementares, incluindo hemograma, avaliação da função renal, dosagem de enzimas cardíacas e radiografia de tórax, não apresentaram anormalidades. O eletrocardiograma demonstrou inversão assimétrica de onda T de V2 a V6 (Figura 1).

Foi realizado tratamento clínico inicial para angina instável com metoprolol, monocordil, heparina de baixo peso molecular, clopidogrel, ácido acetilsalicílico, sinvastatina, captopril e máscara de oxigênio. Momen- 


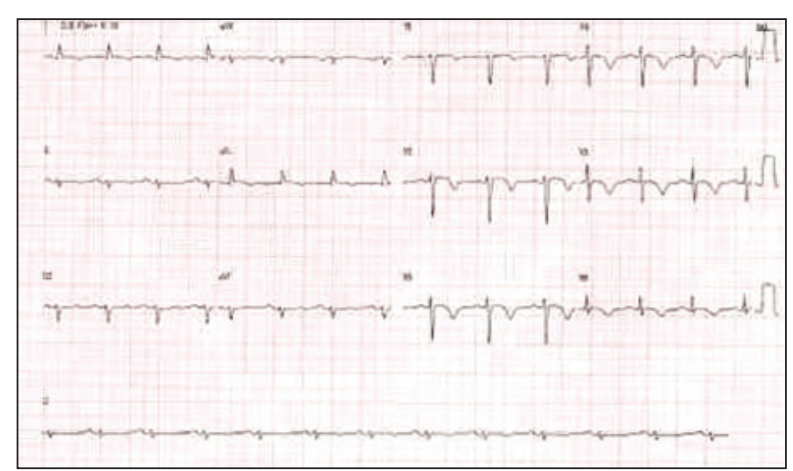

Figura 1 - Eletrocardiograma em ritmo sinusal regular, apresentando discreta inversão de T em D1 e aVL, ausência de progressão de $\mathrm{R}$ em V2 e V3, inversão assimétrica de onda T de V2 a V6 e desvio do eixo SÂQRS para cima e para a esquerda, compatível com bloqueio divisional ântero-superior.

tos após a admissão, a paciente foi encaminhada para estudo hemodinâmico, que evidenciou as seguintes alterações: 1 . tronco da artéria coronária esquerda com lesão grave de $90 \%$ em óstio e aneurisma grave nos terços médio e distal (21 x $16 \mathrm{~mm})$; 2. artéria descendente anterior atingindo o terço distal do sulco interventricular e apresentando lesão ostial de $60 \%$, além de ectasia e trombose proximal, com leito distal de bom calibre; 3. artéria circunflexa isenta de lesões obstrutivas; 4. artéria coronária direita dominante, com irregularidades parietais; e 5. presença de circulação colateral da artéria coronária direita para a descendente anterior de grau II (Figura 2). A ventriculografia revelou ventrículo esquerdo com aspecto hipertrófico e aumento do volume sistólico final decorrente de hipocinesia anterior e apical moderada (Figura 3).

Com base no resultado do cateterismo cardíaco, foram suspensos os medicamentos clopidogrel e ácido acetilsalicílico, e a paciente foi submetida a cirurgia de revascularização do miocárdio em 26 de maio de 2006. Durante o ato cirúrgico, observou-se aterosclerose das artérias torácicas internas (mamárias) e optou-se pelo uso de pontes de veia safena para as artérias descendente anterior e marginal esquerda, ambas com excelente fluxo, e posteriormente ligadura do aneurisma. A paciente evoluiu bem, recebendo alta hospitalar após 12 dias de internação, e permanece assintomática após um ano de acompanhamento ambulatorial.

\section{DISCUSSÃO}

Aneurisma coronário é a presença de um seguimento de artéria coronária com o diâmetro superior a uma vez e meia o diâmetro normal ${ }^{4,5}$. A doença de Kawasaki é a causa mais comum de aneurisma coronário no mundo, especialmente em crianças e mulheres. Nos Estados Unidos, a principal causa é a doença aterosclerótica ${ }^{1}$. Os aneurismas podem ser fusiformes

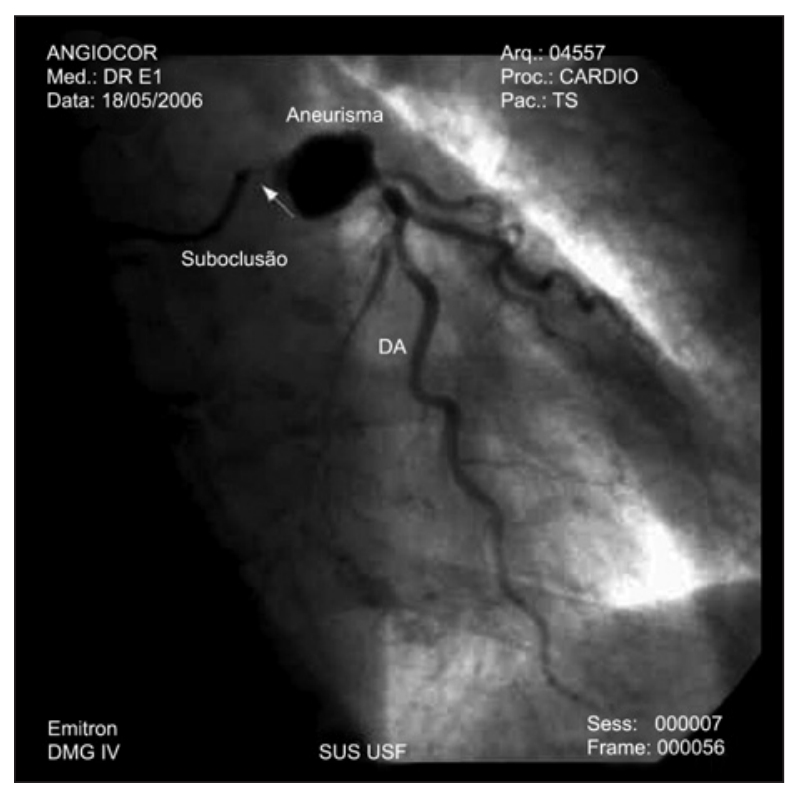

Figura 2 - Coronariografia em póstero-anterior cranial, com tronco da artéria coronária esquerda demonstrando suboclusão ostial e aneurisma acentuado nos terços médio e distal $(21 \times 16 \mathrm{~mm})$. Lesão de $60 \%$ no óstio da artéria descendente anterior com presença de ectasia e trombose proximal. DA = artéria descendente anterior.

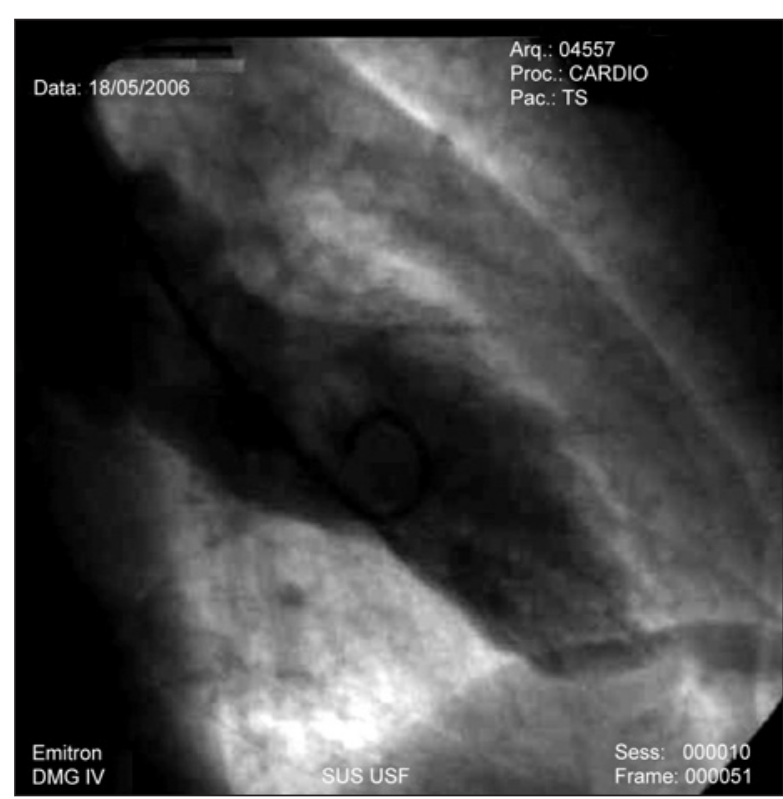

Figura 3 - Ventrículo esquerdo com aspecto hipertrófico e aumento do volume sistólico final decorrente de hipocinesia anterior e apical moderada.

ou saculares ${ }^{6}$, com predominância no sexo masculino, e a incidência varia na literatura entre $0,15 \%$ e $4,9 \%$ em pacientes submetidos a coronariografia ${ }^{2,3}$. A histologia revela hialinização difusa, depósitos de lipídios, ruptura da íntima, calcificação da média, fibrose focal e hemorragia intramural ${ }^{3}$. Essas áreas, 
mesmo se não associadas à estenose, estão sujeitas a espasmos, tromboses, embolias e dissecções espontâneas, que são potenciais causas de síndromes coronarianas agudas ${ }^{7}$.

Os pacientes portadores de aneurismas de artérias coronárias apresentam maior incidência de história familiar de doença coronariana, hipertensão arterial sistêmica, infarto agudo do miocárdio prévio e espasmos, em relação ao grupo controle. Wagdi et al. ${ }^{8}$ fizeram a primeira avaliação de pacientes com aneurisma de coronárias e observaram que pacientes sem doença coronariana obstrutiva, mas com aneurisma, apresentavam maior prevalência de hipertensão arterial. Também encontraram $15 \%$ de mortalidade em dois anos, o que equivale à mortalidade de pacientes com doença coronariana triarterial tratada clinicamente.

Existem poucas descrições cirúrgicas para o tratamento de aneurismas das artérias coronárias, permanecendo controvérsias sobre qual a melhor opção terapêutica ${ }^{9}$. Alguns autores recomendam a revascularização das artérias descendente anterior e circunflexa, com concomitante fechamento distal do segmento dilatado, para prevenir embolização, aumento ou possível ruptura do aneurisma ${ }^{10}$. Foi esse o procedimento realizado com sucesso no caso relatado.

\section{REFERÊNCIAS BIBLIOGRÁFICAS}

1. Guérios EE, Bueno RR, Andrade PM, Nercolini DC, Pacheco $\mathrm{AL}$, Tarastchuk JC, et al. Aneurysm of the left main coronary artery. Arq Bras Cardiol. 2000;75(6):534-6.

2. Swaye PS, Fisher LD, Litwin P, Vignola PA, Judkins MP, Kemp HG, et al. Aneurysmal coronary artery disease. Circulation. 1983;67(1):134-8.

3. Hawkins JW, Vacek JL, Smith GS. Massive aneurysm of the left main coronary artery. Am Heart J. 1990;119(6): 1406-8.

4. Hartnell GG, Parnell BM, Pridie RB. Coronary artery ectasia: its prevalence and clinical significance in 4,993 patients. Br Heart J. 1985;54(4):392-5.

5. Syed M, Lesch M. Coronary artery aneurysm: a review. Prog Cardiovasc Dis. 1997;40(1):77-84.

6. Topaz O, DiSciascio G, Cowley MJ, Goudreau E, Soffer A, Nath $A$, et al. Angiographic features of left main coronary artery aneurysms. Am J Cardiol. 1991;67(13):1139-42.

7. Bove AA, Vlietstra RE. Spasm in ectatic coronary arteries. Mayo Clin Proc. 1985;60(12):822-6.

8. Wagdi P, Vuilliomenet A, Jenzer HR. Left main coronary aneurysm and severe three-vessel disease with sparing of an isolated conus artery. Am Heart J. 1996;131(4):831-3.

9. Lepojarvi M, Salmela E, Huikuri H, Karkola P. Repair of an aneurysm of the left main coronary artery. Ann Thorac Surg. 1996;61(4):1247-9.

10. Fukaya $Y$, Miyakawa M, Senga O, Hikita H, Kouzu S, Tunemoto $\mathrm{H}$. Surgical management of left main coronary artery aneurysm. Ann Thorac Surg. 1994;57(1):228-30. 